Document downloaded from:

http://hdl.handle.net/10251/162570

This paper must be cited as:

Bonet Solves, JA. (2020). A note about the spectrum of composition operators induced by a rotation. Revista de la Real Academia de Ciencias Exactas Físicas y Naturales Serie A Matemáticas. 114(2):1-6. https://doi.org/10.1007/s13398-020-00788-5

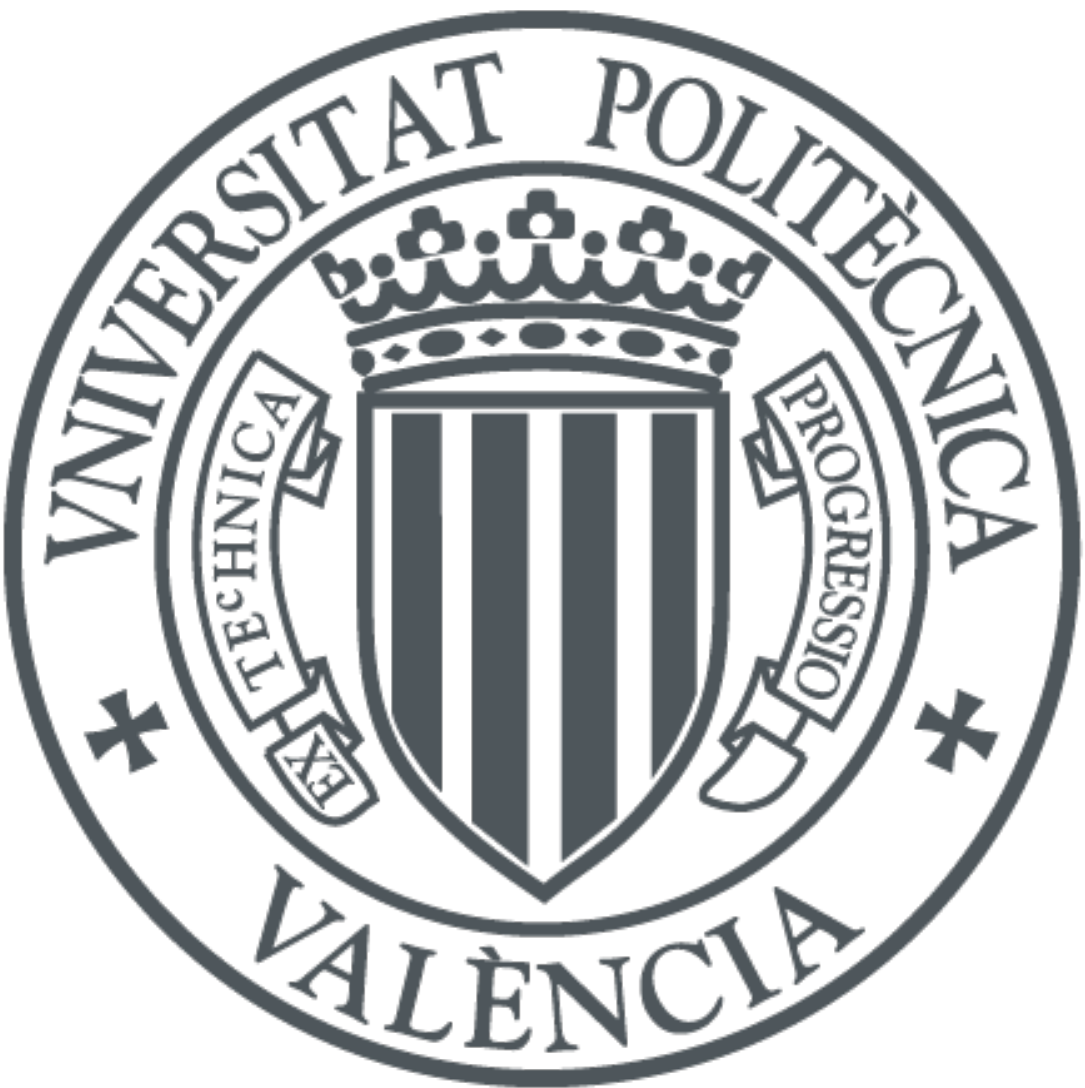

The final publication is available at

https://doi.org/10.1007/s13398-020-00788-5

Copyright Springer-Verlag

Additional Information 


\title{
A note about the spectrum of composition operators induced by a rotation
}

\author{
José Bonet
}

\begin{abstract}
A characterization of those points of the unit circle which belong to the spectrum of a composition operator $C_{\varphi}$, defined by a rotation $\varphi(z)=r z$ with $|r|=1$, on the space $H_{0}(\mathbb{D})$ of all analytic functions on the unit disc which vanish at 0 , is given. Examples show that the spectrum of $C_{\varphi}$ need not be closed. In these examples the spectrum is dense but point 1 may or may not belong to it, and this is related to Diophantine approximation.
\end{abstract}

The aim of this note is to present a few results about the spectrum $\sigma\left(C_{\varphi_{r}}, H_{0}(\mathbb{D})\right)$ of composition operators, defined by a self map of the unit disc $\mathbb{D}$ given by $\varphi_{r}(z):=r z$, for a complex number $r$ with $|r|=1$, when acting on the space $H_{0}(\mathbb{D})$ of all analytic functions on the disc $\mathbb{D}$ vanishing at 0 . Our results complement recent work by Arendt, Celariès and Chalendar [2]. The main result Theorem 2 provides a characterization of the points of the unit circle which belong to the spectrum of a composition operator $C_{\varphi_{r}}$. Moreover, as a consequence of Theorem 4, we show that there exist $r, s \in \mathbb{C}$ with $|r|=|s|=1$, which are not roots of unity, such that the corresponding self-maps $\varphi_{r}$ and $\varphi_{s}$ satisfy $1 \notin \sigma\left(C_{\varphi_{r}}, H_{0}(\mathbb{D})\right)$, in which case $\sigma\left(C_{\varphi_{r}}, H_{0}(\mathbb{D})\right)$ is not closed, and $1 \in \sigma\left(C_{\varphi_{s}}, H_{0}(\mathbb{D})\right)$. The example of $r$ uses Diophantine irrational numbers, see Theorem 4 (1); whereas the example of $s$ as indicated requires a construction given in Lemma 3 of irrational numbers $x$ with a sequence of rational numbers very rapidly converging to $x$ and an argument given in Theorem 4 (2).

Here the space $H(\mathbb{D})$ of all analytic functions on $\mathbb{D}$ is endowed with the complete metrizable locally convex topology of uniform convergence on the compact subsets of $\mathbb{D}$. An analytic selfmap $\varphi$ of $\mathbb{D}$ defines a continuous composition operator $C_{\varphi}: H(\mathbb{D}) \rightarrow H(\mathbb{D}), C_{\varphi}(f):=f \circ \varphi$ for each $f \in H(\mathbb{D})$. The monographs of Cowen and MacCluer [6] and Shapiro [13] are standard references for composition operators on spaces of analytic functions; see also [2], [3], [4], [8], [14] and the references therein.

Let $T: X \rightarrow X$ be a continuous linear operator on a Fréchet space $X$, i.e. a complete metrizable locally convex space. We write $T \in \mathcal{L}(X)$. The resolvent set $\rho(T)$ of $T$ consists of all $\lambda \in \mathbb{C}$ such that $R(\lambda, T):=(\lambda I-T)^{-1}$ is a continuous linear operator, that is $T-\lambda I$ : $X \rightarrow X$ is bijective and has a continuous inverse. Here $I$ stands for the identity operator on $X$. The set $\sigma(T):=\mathbb{C} \backslash \rho(T)$ is called the spectrum of $T$. The point spectrum $\sigma_{p t}(T)$ of $T$ consists of all $\lambda \in \mathbb{C}$ such that $(\lambda I-T)$ is not injective. If we need to stress the space $X$, then we write $\sigma(T ; X), \sigma_{p t}(T ; X)$ and $\rho(T ; X)$. Unlike for Banach spaces $X$, it may happen that $\rho(T)=\emptyset$ or that $\rho(T)$ is not open. This is the reason why some authors (see e.g. [15] and [1]) prefer to consider $\rho^{*}(T)$ instead of $\rho(T)$ consisting of all $\lambda \in \mathbb{C}$ for which there exists $\delta>0$ such that $B(\lambda, \delta):=\{z \in \mathbb{C}:|z-\lambda|<\delta\} \subset \rho(T)$ and $\{R(\mu, T): \mu \in B(\lambda, \delta)\}$ is

2010 Mathematics Subject Classification. Primary: 47B33, secondary: 47A10; 46E10; 11K60

Key words and phrases. Composition operator; space of analytic functions; rotation; Diophantine number 
equicontinuous in $\mathcal{L}(X)$. The Waelbroeck spectrum is defined by $\sigma^{*}(T):=\mathbb{C} \backslash \rho^{*}(T)$. It is a closed set containing $\sigma(T)$. The reader is referred to the book of Meise and Vogt [11] for functional analysis and Fréchet spaces.

Arendt, Celariès and Chalendar [2, Theorem 4.1] prove that if $\varphi: \mathbb{D} \rightarrow \mathbb{D}$ is an analytic map with an interior fixed point $\varphi(a)=a \in \mathbb{D}$ and $0<\left|\varphi^{\prime}(a)\right|<1$, then the spectrum of the composition operator $C_{\varphi}$ is

$$
\sigma\left(C_{\varphi}, H(\mathbb{D})\right)=\sigma^{*}\left(C_{\varphi}, H(\mathbb{D})\right)=\{0\} \cup\left\{\varphi^{\prime}(a)^{n}: n=0,1,2, \ldots\right\} .
$$

Moreover, in case $\varphi^{\prime}(a)=0$, then $\left[2\right.$, Theorem 4.10] implies that $\sigma\left(C_{\varphi}, H(\mathbb{D})\right)=\sigma^{*}\left(C_{\varphi}, H(\mathbb{D})\right)=$ $\{0,1\}$.

The eigenvalues and spectrum of composition operators on Banach spaces of analytic functions has been investigated by many authors. We refer the reader to [2], [3], [8] and [14] and the references therein.

If $\varphi$ is an automorphism with an interior fixed point $\varphi(a)=a \in \mathbb{D}$, then $0 \notin \sigma\left(C_{\varphi}, H(\mathbb{D})\right)$, as follows for example from [2, Corollary 2.4]. Moreover, the Moebius transform $\psi_{a}(z):=$ $(a-z) /(1-\bar{a} z)$ satisfies $\psi_{a}(a)=0$ and $\psi_{a}^{-1}=\psi_{a}$, and

$$
\sigma\left(C_{\psi_{a}} C_{\varphi} C_{\psi_{a}}, H(\mathbb{D})\right)=\sigma\left(C_{\varphi}, H(\mathbb{D})\right) .
$$

Accordingly to investigate the spectrum in this case it is enough to consider the case of rotations $\varphi_{r}(z):=r z, z \in \mathbb{D}$, for each $r \in \mathbb{C}$ with $|r|=1$.

In the rest of the article we denote by $H_{0}(\mathbb{D})$ the space of all the analytic functions on $\mathbb{D}$ such that $f(0)=0$, which is a closed hyperplane of $H(\mathbb{D})$, hence a Fréchet space when endowed with the induced topology. Clearly $C_{\varphi_{r}}\left(H_{0}(\mathbb{D})\right) \subset H_{0}(\mathbb{D})$ for each $|r|=1$. We give several results about the spectrum of the composition operator $C_{\varphi_{r}}$ when acting on $H_{0}(\mathbb{D})$ which complement the aforementioned theorems in [2].

Our first result collects several general facts. Some of them might be known. They are related to the study of the translation operator on the circle, or modulo one, in ergodic theory; see e.g. [7]. From now on we denote by $\mathbb{T}$ the unit circle.

Proposition 1 Let $\varphi_{r}$ be the self map of $\mathbb{D}$ given by $\varphi_{r}(z):=r z, z \in \mathbb{D}$ for some $r \in \mathbb{T}$. Then

(1) $\sigma_{p t}\left(C_{\varphi_{r}}, H(\mathbb{D})\right)=\left\{r^{n} \mid n=0,1,2, \ldots\right\}$.

(2) $\sigma^{*}\left(C_{\varphi_{r}}, H(\mathbb{D})\right)$ is contained in the unit circle $\mathbb{T}$. The same holds for $\sigma^{*}\left(C_{\varphi_{r}}, H_{0}(\mathbb{D})\right)$.

(3) If $r$ is a root of unity and $m$ is the minimum positive integer such that $r^{m}=1$, then

$$
\sigma\left(C_{\varphi_{r}}, H(\mathbb{D})\right)=\sigma^{*}\left(C_{\varphi_{r}}, H(\mathbb{D})\right)=\sigma_{p t}\left(C_{\varphi_{r}}, H(\mathbb{D})\right)=\left\{1, r, r^{2}, \ldots r^{m-1}\right\} .
$$

Moreover $\operatorname{Ker}\left(C_{\varphi_{r}}-r^{j} I\right)$ is infinite dimensional for each $j=0, \ldots, m-1$. The same result holds for $H_{0}(\mathbb{D})$ instead of $H(\mathbb{D})$.

(4) If $r$ is not a root of unity, then $\sigma_{p t}\left(C_{\varphi_{r}}, H_{0}(\mathbb{D})\right)=\left\{r^{n} \mid n=1,2, \ldots\right\}$ and $\overline{\sigma\left(C_{\varphi_{r}}, H_{0}(\mathbb{D})\right)}=$ $\sigma^{*}\left(C_{\varphi_{r}}, H_{0}(\mathbb{D})\right)=\mathbb{T}$. The last equality of sets also holds for $H(\mathbb{D})$. 
Proof. (1) Clearly $C_{\varphi_{r}}\left(z^{n}\right)=r^{n} z^{n}$ for each $n=0,1,2, \ldots$ On the other hand, if $f(z)=$ $\sum_{n=0}^{\infty} a_{n} z^{n} \in H(\mathbb{D}), f \neq 0$, satisfies $f(r z)=\mu f(z)$ for each $z \in \mathbb{D}$, we have $a_{n}\left(r^{n}-\mu\right)=0$ for each $n=0,1,2, \ldots$. Since $f \neq 0$, this implies that $\mu=r^{n}$ for some $n$.

(2) We first show that $C_{\varphi_{r}}-\eta I: H(\mathbb{D}) \rightarrow H(\mathbb{D})$ is bijective for each $\eta \notin \mathbb{T}$, hence an isomorphism by the closed graph theorem. By part (1), $C_{\varphi_{r}}-\eta I$ is injective. We show that it is also surjective. Given $g(z)=\sum_{n=0}^{\infty} a_{n} z^{n} \in H(\mathbb{D})$, define $f(z):=\sum_{n=0}^{\infty} \frac{a_{n}}{r^{n}-\eta} z^{n}$. There is $\delta>0$ such that $\left|\eta-r^{n}\right|>\delta$ for each $n=0,1,2, \ldots$ Since the series $\sum_{n=0}^{\infty}\left|a_{n}\right||z|^{n}$ converges for each $|z|<1$, we get that $\sum_{n=0}^{\infty} \frac{\left|a_{n}\right|}{\left|r^{n}-\eta\right|}|z|^{n}$ also converges for each $|z|<1$, and $f(z)$ is an analytic function on $\mathbb{D}$ such that $\left(C_{\varphi_{r}}-\eta I\right) f=g$.

In particular we have shown that

$$
\left(C_{\varphi_{r}}-\eta I\right)^{-1}\left(\sum_{n=0}^{\infty} a_{n} z^{n}\right)=\sum_{n=0}^{\infty} \frac{a_{n}}{r^{n}-\eta} z^{n} .
$$

for each $\eta \notin \mathbb{T}$ and each $\sum_{n=0}^{\infty} a_{n} z^{n} \in H(\mathbb{D})$.

Now fix $\mu \notin \mathbb{T}$ and select $\delta>0$ such that $|\eta-r|>\delta$ for each $r \in \mathbb{T}$ and each $\eta \in \mathbb{C}$ with $|\eta-\mu|<\delta$. To prove that $\mu \notin \sigma^{*}\left(C_{\varphi_{r}}, H(\mathbb{D})\right)$, by the uniform boundedness principle, it is enough to show that the set

$$
B(g):=\left\{\left(C_{\varphi_{r}}-\eta I\right)^{-1} g|| \eta-\mu \mid<\delta\right\}
$$

is bounded in $H(\mathbb{D})$ for each $g=\sum_{n=0}^{\infty} a_{n} z^{n} \in H(\mathbb{D})$. To see this, fix $\left.\alpha \in\right] 0,1[$. We have

$$
\sup _{|z| \leq \alpha}\left|\left(C_{\varphi_{r}}-\eta I\right)^{-1} g(z)\right|=\sup _{|z| \leq \alpha}\left|\sum_{n=0}^{\infty} \frac{a_{n}}{r^{n}-\eta} z^{n}\right| \leq \delta^{-1} \sum_{n=0}^{\infty}\left|a_{n}\right| \alpha^{n} .
$$

The proof that $\sigma^{*}\left(C_{\varphi_{r}}, H_{0}(\mathbb{D})\right) \subset \mathbb{T}$ is the same.

(3) Clearly

$$
\left\{1, r, r^{2}, \ldots r^{m-1}\right\}=\sigma_{p t}\left(C_{\varphi_{r}}, H(\mathbb{D})\right) \subset \sigma\left(C_{\varphi_{r}}, H(\mathbb{D})\right) \subset \sigma^{*}\left(C_{\varphi_{r}}, H(\mathbb{D})\right) .
$$

The proof each $\mu \notin\left\{1, r, r^{2}, \ldots r^{m-1}\right\}$ does not belong to $\sigma^{*}\left(C_{\varphi_{r}}, H(\mathbb{D})\right)$ is similar to the one in part (2), just taking $\delta>0$ such that $\left|\eta-r^{n}\right|>\delta$ for each $n=0, \ldots, m-1$ and each $\eta \in \mathbb{C}$ with $|\eta-\mu|<\delta$.

The space $\operatorname{Ker}\left(C_{\varphi_{r}}-r^{j} I\right), j=0, \ldots, m-1$, of eigenvectors is infinite dimensional because $C_{\varphi_{r}}\left(z^{k m+j}\right)=r^{j} z^{k m+j}$ for each $k \in \mathbb{N}$.

In the case of $H_{0}(\mathbb{D})$, observe that, although the constants do not belong to this space, we have $C_{\varphi_{r}}\left(z^{k m}\right)=z^{k m}$ for each $k \in \mathbb{N}$. The rest of the proof is similar.

(4) If $r$ is not a root of unity, then $r^{n} \neq 1$ for each $n \in \mathbb{N}$. Since the constants do not belong to $H_{0}(\mathbb{D})$, the argument in part $(1)$ yields $\sigma_{p t}\left(C_{\varphi_{r}}, H_{0}(\mathbb{D})\right)=\left\{r^{n} \mid n \in \mathbb{N}\right\}$.

Therefore, by part (2),

$$
\left\{r^{n} \mid n \in \mathbb{N}\right\} \subset \sigma\left(C_{\varphi_{r}}, H_{0}(\mathbb{D})\right) \subset \sigma^{*}\left(C_{\varphi_{r}}, H_{0}(\mathbb{D})\right) \subset \mathbb{T} .
$$

By Kronecker's Theorem [12, Theorem 2.2.4], which can also be deduced from [9, Theorem 438], $\left\{r^{n} \mid n \in \mathbb{N}\right\}$ is dense in $\mathbb{T}$. This implies

$$
\overline{\sigma\left(C_{\varphi_{r}}, H_{0}(\mathbb{D})\right)}=\sigma^{*}\left(C_{\varphi_{r}}, H_{0}(\mathbb{D})\right)=\mathbb{T} .
$$


The proof that $\sigma^{*}\left(C_{\varphi_{r}}, H(\mathbb{D})\right)=\mathbb{T}$ is obtained with the same argument.

There are examples of continuous linear operators $T$ on a Fréchet space $E$ such that $\overline{\sigma(T, E)}$ is properly contained in $\sigma^{*}(T, E)$. See Remark 3.5 (vi) in [1]. Compare with the statement in Proposition 1 (4).

As a consequence of Proposition 1 (4), if $X$ is a Banach space of analytic functions containing the polynomials which is continuously included in $H(\mathbb{D})$ and such that rotations define continuous composition operators on $X$, then $\sigma\left(C_{\varphi_{r}}, X\right)=\mathbb{T}$. We see below that the behaviour in the Fréchet space $H_{0}(\mathbb{D})$ is different; in particular $\sigma\left(C_{\varphi_{r}}, H_{0}(\mathbb{D})\right)$ need not be closed.

Theorem 2 Let $r \in \mathbb{T}$ be such that $r^{n} \neq 1$ for each $n \in \mathbb{N}$ and let $\varphi_{r}(z):=r z, z \in \mathbb{D}$. Let $\lambda \in \mathbb{T}$ satisfy $\lambda \neq r^{n}$ for each $n \in \mathbb{N}$. The following conditions are equivalent:

(i) $\lambda \notin \sigma\left(C_{\varphi_{r}}, H_{0}(\mathbb{D})\right)$.

(ii) For each $\alpha \in] 0,1\left[\right.$ there exists $\delta(\alpha)>0$ such that $\left|r^{n}-\lambda\right| \geq \delta(\alpha) \alpha^{n}$ for each $n \in \mathbb{N}$.

Proof. By the closed graph theorem, condition (i) holds if and only if $C_{\varphi_{r}}-\lambda I: H_{0}(\mathbb{D}) \rightarrow$ $H_{0}(\mathbb{D})$ is bijective. Since $\lambda \neq r^{n}$ for each $n \in \mathbb{N}, C_{\varphi_{r}}-\lambda I$ is injective by Proposition 1.

(ii) implies (i). It is enough to show that condition (ii) implies that $C_{\varphi_{r}}-\lambda I: H_{0}(\mathbb{D}) \rightarrow$ $H_{0}(\mathbb{D})$ is surjective. Fix $g(z)=\sum_{n=1}^{\infty} a_{n} z^{n} \in H_{0}(\mathbb{D})$ and define $f(z):=\sum_{n=1}^{\infty} \frac{a_{n}}{r^{n}-\lambda} z^{n}$. Fix $\alpha \in] 0,1[$ and select $\beta \in] \alpha, 1\left[\right.$. By (ii), there is $\delta(\beta)>0$ such that $\left|r^{n}-\lambda\right| \geq \delta(\beta) \beta^{n}$ for each $n \in \mathbb{N}$. For each $|z| \leq \alpha$ we have

$$
\sum_{n=1}^{\infty} \frac{\left|a_{n}\right|}{\left|r^{n}-\lambda\right|}|z|^{n} \leq \frac{1}{\delta(\beta)} \sum_{n=1}^{\infty}\left|a_{n}\right| \frac{\alpha^{n}}{\beta^{n}}<\infty
$$

Therefore $f \in H_{0}(\mathbb{D})$ and, clearly, $\left(C_{\varphi_{r}}-\lambda I\right) f=g$.

(i) implies (ii). If $\lambda \notin \sigma\left(C_{\varphi_{r}}, H_{0}(\mathbb{D})\right)$, then $C_{\varphi_{r}}-\lambda I: H_{0}(\mathbb{D}) \rightarrow H_{0}(\mathbb{D})$ is surjective. Given $\sum_{n=1}^{\infty} z^{n} \in H_{0}(\mathbb{D})$, its pre-image by $C_{\varphi_{r}}-\lambda I$ is $\sum_{n=1}^{\infty} \frac{1}{r^{n}-\lambda} z^{n}$ and it belongs to $H_{0}(\mathbb{D})$. In particular, for each $\alpha \in] 0,1[$,

$$
\sum_{n=1}^{\infty} \frac{1}{\left|r^{n}-\lambda\right|} \alpha^{n}<\infty .
$$

Therefore $\sup _{n \in \mathbb{N}} \frac{1}{\left|r^{n}-\lambda\right|} \alpha^{n}<\infty$, which clearly implies (ii).

A real number $x \in \mathbb{R}$ is called Diophantine if there are $\delta \geq 1$ and $c(x)>0$ such that

$$
\left|x-\frac{p}{q}\right| \geq \frac{c(x)}{q^{1+\delta}}
$$

for all rational numbers $p / q$. See [12, Definition 3.1.9]. The complement of the set of Diophantine numbers has Lebesgue measure 0; see [5, page 43]. By a Theorem of Liouville (see [9, Theorem 191] or [12, Proposition 3.1.3]) every irrational algebraic number of degree larger than or equal 2 is Diophantine. See also [10] for further information.

The proof of Theorem 4 below requires the construction of irrational numbers $x$ with very fast Diophantine approximation. The next lemma follows from the more general result Theorem 22 in [10]. We include a proof which does not use continued fractions for the sake of completeness. 
Lemma 3 For each $m \in \mathbb{N}, m \geq 2$, there is a positive real number $x(m)$ and sequences of positive numbers $\left(p_{j}\right)_{j=1}^{\infty}$ and $\left(q_{j}\right)_{j=1}^{\infty}$ with $\lim _{j \rightarrow \infty} q_{j}=+\infty$ such that

$$
\left|x(m)-\frac{p_{j}}{q_{j}}\right| \leq\left(\frac{1}{m}\right)^{q_{j}}
$$

Proof. Given $m \in \mathbb{N}, m \geq 2$, define inductively $q_{1}:=m$ and $q_{j+1}:=\left(q_{j}\right)^{q_{j}}$ for $j \in \mathbb{N}$. Clearly $q_{j} \geq m^{j}$ and $q_{j}$ is a divisor of $q_{j+1}$ for each $j \in \mathbb{N}$. We set $x(m):=\sum_{j=1}^{\infty} 1 / q_{j}$. The series converges, and each partial sum $\sum_{j=1}^{k} 1 / q_{j}$ is a rational number which can be written of the form $p_{k} / q_{k}$ for some $p_{k} \in \mathbb{N}$. Moreover, we have

$$
\left|x(m)-\frac{p_{k}}{q_{k}}\right|=\sum_{j=k+1}^{\infty} \frac{1}{q_{j}} \leq \frac{1}{q_{k+1}} \sum_{j=0}^{\infty}\left(\frac{1}{m}\right)^{j} \leq \frac{2}{q_{k+1}} .
$$

Since $2 m^{q_{k}} \leq q_{k+1}$ for each $k \in \mathbb{N}$, we have $2 / q_{k+1} \leq(1 / m)^{q_{k}}$ for each $k \in \mathbb{N}$. Therefore the rational numbers $p_{k} / q_{k}, k \in \mathbb{N}$, satisfy

$$
\left|x(m)-\frac{p_{k}}{q_{k}}\right| \leq\left(\frac{1}{m}\right)^{q_{k}}
$$

for all $k \in \mathbb{N}$.

Theorem 4 (1) If $x \in \mathbb{R}$ is a Diophantine number, then the rotation $\varphi_{r}(z)=r z, z \in \mathbb{D}$, for $r:=e^{i 2 \pi x}$ satisfies that $1 \notin \sigma\left(C_{\varphi_{r}}, H_{0}(\mathbb{D})\right)$. In particular, $1 \in \sigma^{*}\left(C_{\varphi_{r}}, H_{0}(\mathbb{D})\right) \backslash \sigma\left(C_{\varphi_{r}}, H_{0}(\mathbb{D})\right)$, and $\sigma\left(C_{\varphi_{r}}, H_{0}(\mathbb{D})\right)$ is not closed.

(2) Let $x \in \mathbb{R}$ be an irrational number such that there are $\alpha \in] 0,1[$ and a sequence of rational numbers $\left(p_{j} / q_{j}\right)_{j=1}^{\infty}$ with $\lim _{j \rightarrow \infty} q_{j}=+\infty$ such that

$$
\left|x-\frac{p_{j}}{q_{j}}\right| \leq \alpha^{q_{j}}
$$

for each $j \in \mathbb{N}$. Then the rotation $\varphi_{r}(z):=r z, z \in \mathbb{D}$, for $r:=e^{i 2 \pi x}$ satisfies $1 \in \sigma\left(C_{\varphi_{r}}, H_{0}(\mathbb{D})\right)$.

Proof. (1) If $x$ is a Diophantine number, then there are $d>0$ and $\delta \geq 1$ such that $r:=e^{i 2 \pi x}$ satisfies $\left|r^{n}-1\right| \geq d / n^{\delta}$ for all $n \in \mathbb{N}$, [5, page 43]. Hence there is $D>0$ such that $1 /\left|r^{n}-1\right| \leq D n^{\delta}$ for each $n \in \mathbb{N}$. Given $\left.\alpha \in\right] 0,1[$, for each $n \in \mathbb{N}$, we have

$$
\frac{1}{\left|r^{n}-1\right|} \alpha^{n} \leq D n^{\delta} \alpha^{n}
$$

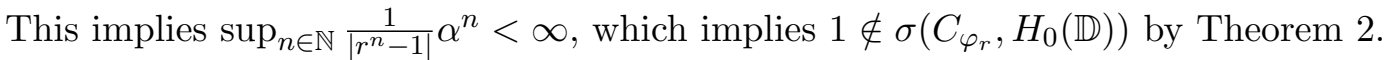

The spectrum $\sigma\left(C_{\varphi_{r}}, H_{0}(\mathbb{D})\right)$ is not closed in this case, because it contains $\left\{r^{n} \mid n \in \mathbb{N}\right\}$, which is dense in $\mathbb{T}$ by Kronecker's Theorem, but $1 \notin \sigma\left(C_{\varphi_{r}}, H_{0}(\mathbb{D})\right)$.

(2) The complex number $r:=e^{i 2 \pi x}$ satisfies, for each $j \in \mathbb{N}$,

$$
\left|r^{q_{j}}-1\right|=\left|e^{i 2 \pi q_{j} x}-e^{i 2 \pi p_{j}}\right| \leq 2 \pi\left|q_{j} x-p_{j}\right| \leq 2 \pi q_{j} \alpha^{q_{j}} .
$$

Proceeding by contradiction, assume that $1 \notin \sigma\left(C_{\varphi_{r}}, H_{0}(\mathbb{D})\right)$. Select any $\left.\beta \in\right] \alpha, 1[$. By Theorem 2, there is $\delta(\beta)>0$ such that $\left|r^{n}-1\right| \geq \delta(\beta) \beta^{n}$ for each $n \in \mathbb{N}$. Specializing for $n=q_{j}, j \in \mathbb{N}$, we get

$$
\delta(\beta) \beta^{q_{j}} \leq\left|r^{q_{j}}-1\right| \leq 2 \pi q_{j} \alpha^{q_{j}} \text { for each } j \in \mathbb{N} .
$$


This is a contradiction, since $0<\alpha<\beta$ and $q_{j}$ tends to infinity as $j$ goes to infinity.

Corollary 5 There exist $r, s \in \mathbb{T}$ such that the corresponding rotations $\varphi_{r}$ and $\varphi_{s}$ satisfy $1 \notin \sigma\left(C_{\varphi_{r}}, H_{0}(\mathbb{D})\right)$ and $1 \in \sigma\left(C_{\varphi_{s}}, H_{0}(\mathbb{D})\right)$.

Proof. This is a direct consequence of Theorem 4 since Lemma 3 ensures the existence of irrational numbers satisfying the hypothesis of part (2) in the Theorem.

Acknowledgement. The author is grateful to J.L. Varona for providing him with useful references about number theory.

The research of this paper was partially supported by the projects MTM2016-76647-P and GV Prometeo/2017/102.

\section{References}

[1] A.A. Albanese, J. Bonet, W.J. Ricker, Montel resolvents and uniformly mean ergodic semigroups of linear operators, Quaest. Math. 36 (2013), 253-290.

[2] W. Arendt, B. Celariès, I. Chalendar, In Koenigs' footsteps: diagonalization of composition operators, arXiv: 1903.04990v1.

[3] R. Aron, M. Lindström, Spectra of weighted composition operators on weighted Banach spaces of analytic functions, Israel J. Math. 141 (2004), 263-276.

[4] J. Bonet, P. Domanski, A note on mean ergodic composition operators on spaces of holomorphic functions. Rev. R. Acad. Cienc. Exactas Fís. Nat. Ser. A Mat. RACSAM 105 (2011), 389-396.

[5] L. Carleson, T.W. Gamelin, Complex Dynamics, Springer, New York, 1993.

[6] C. Cowen, B. MacCluer, Composition Operators on Spaces of Analytic Functions, CRC Press, Boca Raton, FL, 1995.

[7] T. Eisner, B. Farkas, M. Haase, R. Nagel, Operator Theoretic Aspects of Ergodic Theory, Graduate Texts in Mathematics 272, Springer, New York, 2015.

[8] T. Eklund, M. Lindstrm, P. Mleczko, M. Rzeczkowski, Spectra of weighted composition operators on abstract Hardy spaces, Rev. R. Acad. Cienc. Exactas Fís. Nat. Ser. A Mat. RACSAM 113 (2019), 267-279.

[9] G.H. Hardy, E.M. Wright, An Introduction to the Theory of Numbers, Sixth Edition, Oxford University Press, Oxford, 2008.

[10] A. Ya. Khinchin, Continued fractions. Translated from the third (1961) Russian edition. Reprint of the 1964 translation. Dover Publications, Inc., Mineola, NY, 1997.

[11] R. Meise and D. Vogt.: Introduction to Functional Analysis, The Clarendon Press Oxford University Press, New York, 1997.

[12] H. Queffélec, M. Queffélec: Diophantine Approximation and Dirichlet series, Hindustain Book Agency, New Delhi, 2013. 
[13] J.H. Shapiro, Composition Operators and Classical Function Theory, New York, Springer, 1993.

[14] J.H. Shapiro, Composition operators and Schröder's functional equation, Contemporary Math. 213 (1998), 213-228.

[15] F. H Vasilescu, Analytic functional calculus and spectral decompositions. Translated from the Romanian. Mathematics and its Applications (East European Series), 1. D. Reidel Publishing Co., Dordrecht, 1982.

\section{Author's address:}

Instituto Universitario de Matemática Pura y Aplicada IUMPA, Universitat Politècnica de València, E-46071 Valencia, SPAIN

email:jbonet@mat.upv.es 